\title{
General index
}

Abraham, Nicolas, and Maria Torok 85

Adorno, Theodor 21, 37-8, 42

Agrippa, Heinrich Cornelius 220

Aiken, Conrad 15 n.2

Andrewes, Lancelot 183

Attridge, Derek 109-10

Auden, W. H. 102, 129

Augustine 24

Barthes, Roland 21

Beckett, Samuel 3, 6, 92, 120, 153-7, 160-9, $172,181 \mathrm{n} .77,183$

Bellow, Saul 15 n.2

Benjamin, Walter 42

Bennett, Andrew, and Nicholas Royle 3, 127 n. 21

Berger, Anne 115 n.15

Bevington, David 45

Blake, William 4, 15 n.4, 24, 49, 52 n.28

Blanchot, Maurice 21, 226 n.37

Bloom, Harold 69

Blunden, Vicky 185

Bonaparte, Marie 173, 181 n.76

Bonaparte, Napoleon 153

Boswell, James 213

Bowen, Elizabeth 6-8, 10, 80, 122, 127 n.21, 223 n.6

Brahic, Beverley Bie 143 n.30, 216-17, 222

Brontë, Emily 6-7, 10, 24, 62, 70-5, 77 n.20, $80,166,197$

Brooks, Harold 141

Browne, Thomas 152

Browning, Robert 208

Büchner, Karl Georg 24

Busch, Wilhelm 5
Carroll, Lewis 6, 9, 28, 109-13, 117-25, $131-2,172,208$

Caruth, Cathy 29

Carver, Raymond 7

Celan, Paul 170

Cervantes, Miguel de 41

Chaucer, Geoffrey 162

Chekhov, Anton 104

Christie, Agatha 198-201

Clark, Timothy 180 n.66, 208, 210

Cohen, Josh 42

Colebrook, Claire 180 n.66

Coleridge, Samuel Taylor 65

Compton-Burnett, Ivy 15 n.2

Conrad, Joseph 80, 223 n.6

Cornell, Sarah 119

Currie, Mark 57

Dante Alighieri 3, 219

da Vinci, Leonardo 13

de Bono, Edward 158

Deleuze, Gilles 1, 21, 58, 82, 92-3, 167, 168

DeLillo, Don 223 n.6

de Man, Paul 95-6

Derrida, Jacques 1, 13, 21, 27-8, 56-9, 85, 104-13, 115 n.11, 115 n.12, 115 n.15, $159,165-6,188,210$

The Animal That Therefore I Am 217

'Ants' 3, 68-9, 174, 175

The Beast and the Sovereign (vol. 2) 57

'Cinema and Its Ghosts' 212

'Countersignature' 113

'The Double Session' 105

'Fichus' 37-9, 42, 48-9, 137-8, 147

For What Tomorrow 130, 131, 133-4, 138

'Force and Signification' 27 
Derrida, Jacques (cont.)

'Fors' 207

'Freud and the Scene of Writing' 130

Geneses, Genealogies, Genres and Genius $41,136-7,138,171$

Glas 78-9

H. C. for Life, That Is to Say... 7, 8, 40, 79, $118,130,138-9,154,156,157,161-4$, $166,167,170,173,174,178$ n.30, 184, 190-1, 194, 195, 225 n.16

'Living On: Border Lines' 193

Monolingualism of the Other 27, 138

Of Grammatology 137, 177 n.24

'Rams' 170

'Resistances' 150

'Shibboleth' 180 n.62

'A Silkworm of One's Own' 103, 105

Sovereignties in Question 119

Spectres of Marx 129, 185, 194, 197

'Telepathy' 72, 105, 137, 172-5, 183

'This Strange Institution Called Literature' 194

'The Time is Out of Joint' 185

'To Speculate - On "Freud"' 39-40, 128, 134-5

Dickinson, Emily 65, 129

Donne, John 24, 60 n.21

Dostoevsky, Fyodor 28, 80, 148

Doyle, Arthur Conan 173

Duras, Marguerite 28

Dutoit, Thomas 153

Eliot, George 152, 173

Eliot, T. S. 183

Ellis, Havelock 134

Faulkner, William 28, 80

Fliess, Wilhelm 173

Foucault, Michel 1, 21

Freud, Sigmund 9-10, 26-8, 70, 72, 101, 123, 134-6, 145-50, 172-5, 178 n.26, 196

'Difficulties and First Approaches' 136

'A Difficulty in the Path of Psychoanalysis' 27

'A Disturbance of Memory on the Acropolis' 147

The Interpretation of Dreams 41-2, 129-30, 132, 147

Jokes and Their Relation to the

Unconscious 4, 41

'Medusa's Head' 121
'My Contact with Joseph Popper-Lynkeus' 132-3

'Negation' 42

'A Note on the Unconscious in Psychoanalysis' 148-50

'The Question of Lay Analysis' 27, 128

'To Thomas Mann on his Sixtieth Birthday' 27

'The Uncanny' 54-5, 106-7, 119

Fuentes, Carlos 24, 49-50

Genet, Jean 28, 78-9, 82, 103, 221

Golding, William 15 n.2

Haggard, Henry Rider 173

Hamsun, Knut 80

Hanrahan, Mairéad 177 n.25, 224 n.1

Hardy, Thomas 2-3, 173, 208

Haworth, Michael 181 n.81

Heine, Heinrich 41

Hemingway, Ernest 7

Hibbard, G. R. 170

Holbein, Hans 22

Irigaray, Luce 21

Jackson, Richard 213

Jackson, Sarah 22

Jacobson, Howard 146

James, Henry 118, 165, 191, 213

Jensen, Wilhelm 145-6

Johnson, Samuel 213

Jones, Ernest 134-5

Joyce, James 1, 3, 6, 15 n.2, 28, 120, 189, 208

A Portrait of the Artist as a Young Man 8, 93, 219-20

Ulysses 36, 219-20

Kafka, Franz 28, 47, 57, 79-80, 90, 120, 227-8 n.38

Kamuf, Peggy 218, 226 n.20

Keats, John 94-7, 140, 152

Kipling, Rudyard $15 \mathrm{n} .2$

Kleist, Heinrich von 28, 221

Kristeva, Julia 21

Lacan, Jacques 1, 21, 28, 192

Lacey, Candida 185

Lardner, Ring 155

Larsen, Nella 80

Laurie, Hugh 146 
Lawrence, D. H. 80, 206 n.45

Lecercle, Jean-Jacques 116 n.21

Lispector, Clarice 1, 7, 28, 57, 80, 118, 120, 166-7, 221, 228 n.39

Love, Brenda 189

Lubbock, Percy 213

Lyotard, Jean-François 21, 56, 60 n.18

Mandelstam, Osip 28

Marder, Elissa 86 n.6, 221, 229 n.63

Marvell, Andrew 163

Mayoux, Jean-Jacques 8, 15 n.32

McEachern, Claire 50 n.2

McGregor, Jon 223 n.6

McGurl, Mark 21, 192

Melville, Herman 80

Meyers, F. W. H. 173

Michaud, Ginette 156

Milesi, Laurent 171

Milton, John 120

Montaigne, Michel de 28, 47, 152

Morrison, Toni 80

Morton, Timothy 180 n.66, 209

Murray, Les 98 n.7

Nietzsche, Friedrich 22, 39

Ovid 5, 28, 56, 81

Pearlman, Corinne 185

Phillips, Adam 9-10, 26

Picasso, Pablo 13, 92

Piles, Roger de 31 n.17

Plath, Sylvia 56

Plato 84-5

Poe, Edgar Allan 6, 9, 10, 28, 30, 62-4, 102, 103 n.5, 166

Popper-Lynkeus, Joseph 132-3

Pound, Ezra 15 n.2

Prenowitz, Eric 76-7 n.18, 178 n.37

Proust, Marcel 47, 207, 208

Reid, Iain 199

Rembrandt van Rijn 12-14, 22-3

Rimbaud, Arthur 4, 28

Roe, John 198

Sellers, Susan 119

Shakespeare, William 1, 6, 9, 10, 26, 28
Antony and Cleopatra 26, 43-50, 96-7, 129, 142, 170, 206 n.45

As You Like It 26

Hamlet 6, 22, 26, 113, 135, 159-60, 161, 168, 169-70, 195, 198, 206 n.45

King Lear 26, 139, 198

Love's Labour's Lost 219-20

Macbeth 26, 132, 170, 188, 196

Measure for Measure $206 \mathrm{n} .46$

A Midsummer Night's Dream 26, 62, 66-7,

76 n.10, 76 n.11, 135-6, 139-41

Much Ado About Nothing 35-6

Othello 26

The Rape of Lucrece 197-8

Richard III 184-5, 195

Romeo and Juliet 29-30, 41, 42, 65, 120, $128-9,213,216,221$

Sonnets 58, 160, 206 n.46

The Tempest 2, 4, 132

Twelfth Night 54

Shelley, Percy Bysshe 62, 63, 140

Sidney, Philip 45

Smith, Nick 105 n.44

Smith, Robert Rowland 188

Sophocles 41

Spark, Muriel 15 n.2

Stendhal 1, 24, 28, 69

Stevens, Wallace $24,85,92-3,146,195$, 216

Stewart, Garrett 189

Still, Judith 22

Strachey, James 142 n.17

Swedenborg, Emanuel 220

Sweet, Henry 186

Thatcher, Margaret 153

Torok, Maria, and Nicolas Abraham 85

Trump, Donald 146, 150

Tsvetaeva, Marina 28, 57, 83, 120

Verne, Jules 41

Wilders, John 45

Wolfe, Cary 227 n. 35

Wood, Sarah 180 n.66, 196

Woolf, Virginia 63, 67-9, 208

Wordsworth, William 4, 67 n.11, 198, 208

Yeats, W. B. 216 